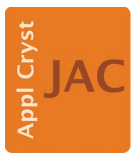

JOURNAL OF

APPLIED

CRYSTALLOGRAPHY

ISSN 1600-5767

Received 27 July 2016

Accepted 26 November 2016

Edited by K. Chapman, Argonne National

Laboratory, USA

Keywords: X-ray diffraction; stress measurement; area detectors; portable X-ray devices.

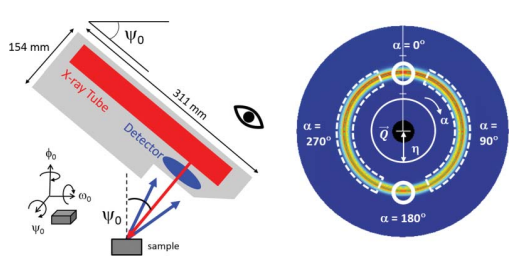

C 2017 International Union of Crystallography

\section{Precision and accuracy of stress measurement with a portable $X$-ray machine using an area detector}

\author{
Seung-Yub Lee, ${ }^{a}$ Jinjing Ling, ${ }^{a}$ Shenghe Wang ${ }^{a}$ and Joaquin Ramirez-Rico ${ }^{b} *$ \\ aApplied Physics and Applied Mathematics, Columbia University, New York, NY 10027, USA, and ${ }^{\mathbf{b}}$ Fisica de la Materia \\ Condensada - ICMS, Universidad de Sevilla - CSIC, 41012 Sevilla, Spain. *Correspondence e-mail: jrr@us.es
}

The use of portable X-ray stress analyzers, which utilize an area detector along with the newly adopted ' $\cos \alpha$ ' or full-ring fitting method, has recently attracted increasing interest. In laboratory conditions, these measurements are fast, convenient and precise because they employ a single-exposure technique that does not require sample rotation. In addition, the effects of grain size and orientation can be evaluated from the Debye ring recorded on the area detector prior to data analysis. The accuracy of the measured stress, however, has been questioned because in most cases just a single reflection is analyzed and the sample-to-detector distances are relatively short. This article presents a comprehensive analysis of the uncertainty associated with a state-of-the-art commercial portable X-ray device. Annealed ferrite reference powders were used to quantify the instrument precision, and the accuracy of the stress measurement was tested by in situ tensile loading on 1018 carbon steel and 6061 aluminium alloy bar samples. The results show that the precision and accuracy are sensitive to the instrument (or sample) tilt angle $\left(\psi_{0}\right)$ as well as to the selected $h k l$ reflection of the sample. The instrument, sample and data analysis methods all affect the overall uncertainty, and each contribution is described for this specific portable X-ray system. Finally, on the basis of the conclusions reached, desirable measurement/analysis protocols for accurate stress assessments are also presented.

\section{Introduction}

X-ray diffraction techniques have been used to determine the near-surface stress state of crystalline materials for nearly a century, and significant advances have been made in both hardware technologies and analysis methods (Noyan \& Cohen, 1987). The former includes high-resolution detectors, modern optical devices and associated electronics. The latter corresponds to the advanced grain interaction models (Kroner, 1958; Hashin \& Shtrikman, 1962; Dölle, 1979), as well as to the integration of finite element simulations (Chen \& Kovacevic, 2003; Clausen, Lee et al., 2003). High precision and accuracy of the measured stress must be balanced with other important criteria, such as measurement speed and portability for industrial applications. Formerly, small devices with portable films provided less reliable results compared to a laboratory diffractometer, but advances in the reliability of portable equipment have opened the door to in situ and in-line measurements, which are of great industrial interest.

The idea of miniaturization of the X-ray system itself is not new at all. Early reports of portable devices from the 1960 s described residual stress measurement in aircraft parts using back-reflection photographic film techniques (Bolstad, 1967; Homicz, 1967). Later generations of portable devices (James \& Cohen, 1978a; Ruud et al., 1984; Araki, 1989; Brauss et al., 1996; Monin et al., 2003; Farrell, 2010) utilized position 


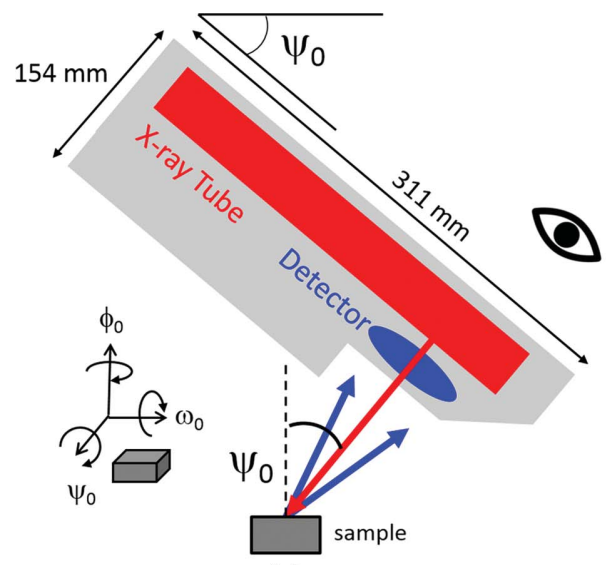

(a)

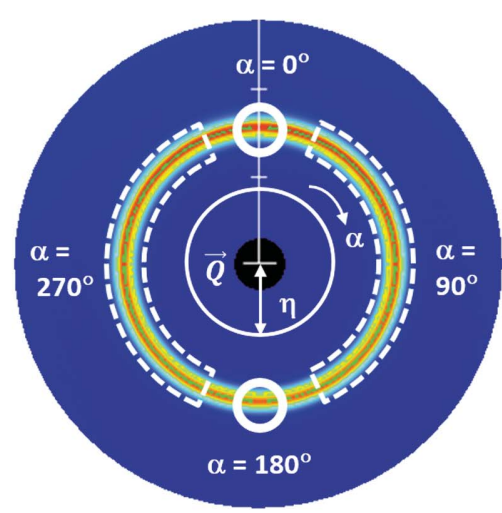

(b)

Figure 1

(a) A schematic diagram of the portable X-ray device used in this study. The detector (shaded in blue) has a diameter of $60 \mathrm{~mm}$ with a $6 \mathrm{~mm}$ hole at the center for beam penetration. (b) A typical diffraction pattern of Fe 211 viewed from the top. Data used for the $\sin ^{2} \psi$ and $\cos \alpha$ methods are marked with hollow circles and dashed lines, respectively.

sensitive detectors (PSDs) to minimize or completely remove detector rotation because non-digitized film reading is laborious (Monin et al., 2000) and any motion of sample or detector can introduce significant errors due to the short sample-todetector distance. For example, the double exposure technique is ten times more sensitive to the displacement error than a single exposure (Ruud et al., 1984). Two-dimensional image plates, also known as storage phosphors, were developed in the 1980s, first for diagnostic radiography (Sonoda et al., 1983) and later for X-ray diffraction (Miyahara et al., 1986; Amemiya \& Miyahara, 1988; Yoshioka \& Ohya, 1992). This laser-stimulated luminescence technique replaced the old films and even one-dimensional PSDs in portable X-ray systems. Other types of area detectors, such as two-dimensional PSDs (multi-wire PSDs) and charge coupled devices (CCDs), also competed with the image plate. Detailed descriptions of the advantages and disadvantages of each detector (Eathough $e t$ al., 1999) and a performance comparison between image plates and CCDs (Kiss et al., 2002) can be found elsewhere.

Another critical component in the success of new portable devices utilizing area detectors is the development of stress determination methods that take full advantage of what has been termed 'two-dimensional' diffraction: $\cos \alpha$ (Taira et al., 1978; Taira \& Tanaka, 1979), $\mathrm{XRD}^{2}(\mathrm{He}, 2011)$ and full-ring fitting methods (Kampfe et al., 2000), ${ }^{1}$ all of which make use of the contour of the Debye ring. Regardless of detector type, the $\sin ^{2} \psi$ method was ubiquitous until the 1980s; since the 1990s, two-dimensional methods have become more available along with high-resolution area detectors.

Currently, there are many competing efforts developing high-resolution portable X-ray devices ${ }^{2}$ for industrial applications. Initially these were targeted for the steel industry

\footnotetext{
${ }^{1} \mathrm{XRD}^{2}$ and the full-ring fitting method are basically the same, but only part of the ring is used in the $\mathrm{XRD}^{2}$ system.

${ }^{2} \mu$-X360 (Pulstec), SmartSite RS (Rigaku), mXRD (Proto), Xstress 3000 (Stressteck), MAX (TEC), Xsolo (Inel) etc. are currently available portable Xray devices for stress measurements.
}

(Ganesh et al., 2013; Farrell, 2010), but applications are expanding alongside the shrinking scale of functional materials. Despite the obvious benefits of a portable device, there have been no objective evaluations of the uncertainty budget of advanced, commercial, portable X-ray units from third parties without conflict of interest. Furthermore, there has been no agreement among experts about the most efficient, precise and accurate stress determination methods. For these reasons, we chose one of the state-of-the-art portable X-ray devices equipped with an image plate and carried out a series of systematic tests on both precision and accuracy of the stress measurement.

A schematic of the portable device used in this study is displayed in Fig. 1(a), and a typical Fe 211 powder pattern is shown in Fig. 1(b). We previously reported that the strain precision of this portable machine is about 9 microstrain $(\mu \varepsilon)$ with ferrite reference powders (Ling \& Lee, 2015), and that in essence the $\sin ^{2} \psi$ and $\cos \alpha$ methods are theoretically identical (Ramirez-Rico et al., 2016) and yield statistically indistinguishable experimental results (Ramirez-Rico et al., 2016; Ling \& Lee, 2015). The mathematical equivalency between $\sin ^{2} \psi$ and $\cos \alpha$ was also shown very recently (Miyazaki \& Sasaki, 2016). Stress measurement with an image plate and the $\cos \alpha$ method is now known to be fast (90 s for steel), convenient (no center of rotation alignment) and precise (2 $\mathrm{MPa}$ repeatability in ferritic steel), in a well controlled environment. However, measured stress values often differ from the known applied ones, and only a narrow range of measurement conditions have been tested. Thus it is necessary to investigate measurement accuracy in more relaxed environments that resemble field or production line conditions.

In this article, we report comprehensive uncertainty analyses of a portable X-ray device, including the measurement parameter dependency of both stress precision (\$4.1) and accuracy ( $\$ 4.2)$, as well as the effect of the choice of a particular reflection (\$4.3). Uncertainties from each error source are discussed in $\S 5$.

\section{Stress determination methods}

Diffraction-based stress determination is an indirect conversion process from a strain, $\varepsilon$, to a stress, $\sigma$, tensor via a constitutive law such as

$$
\sigma_{i j}=C_{i j k l} \varepsilon_{k l},
$$

where $C_{i j k l}$ are the components of the elastic stiffness tensor. If a material is elastically isotropic, equation (1) can be simplified to 


$$
\varepsilon_{i j}=\frac{1+v}{E} \sigma_{i j}-\delta_{i j} \frac{v}{E} \sigma_{k k},
$$

with two independent parameters, Young's modulus $E$ and Poisson's ratio $v$. Here $\delta_{i j}=0$ if $i \neq j$ or $\delta_{i j}=1$ if $i=j$. The interplanar spacing $d_{h k l}$ is used as a built-in strain gauge and calculated from the diffraction peak position in the laboratory coordinate system $\left(\psi_{0}, \varphi_{0}\right)$; corresponding strains are transformed into the sample coordinates $(\psi, \varphi)$ before stress conversion. Starting from the basic formula of equation (2), the next two sub-sections briefly describe the equations used in the $\sin ^{2} \psi$ and $\cos \alpha$ methods.

\subsection{The $\sin ^{2} \psi$ method}

The strain measured along the scattering vector at angles $(\psi, \varphi)$ from the $z$ and $x$ axes can be written in terms of the strain component $\varepsilon_{i j}$ in the sample coordinate system as

$$
\begin{aligned}
\varepsilon_{\psi \varphi}= & \frac{d_{\psi \varphi}-d_{0}}{d_{0}}=\left(\varepsilon_{11} \cos ^{2} \varphi+\varepsilon_{12} \sin 2 \varphi+\varepsilon_{22} \sin ^{2} \varphi-\varepsilon_{33}\right) \\
& \times \sin ^{2} \psi+\varepsilon_{33}
\end{aligned}
$$

by assuming a bi-axial plane stress condition without steep stress gradients in the near-surface region. Here $d_{\psi \varphi}$ is the interplanar spacing at $\psi$ and $\varphi$, and $d_{0}$ is the unstressed interplanar spacing. If the material's elastic behavior is isotropic and homogeneous, equation (3) can be expressed in terms of stress via equation (2), as follows:

$$
\frac{d_{\psi \varphi}-d_{0}}{d_{0}}=\frac{1+v}{E} \sigma_{\varphi} \sin ^{2} \psi-\frac{v}{E}\left(\sigma_{11}+\sigma_{22}\right),
$$

where $\sigma_{\varphi}=\sigma_{11} \cos ^{2} \varphi+\sigma_{12} \sin 2 \varphi+\sigma_{22} \sin ^{2} \varphi$.

If the in-plane stress is not dependent on angle $\varphi$, the equation can be further simplified as

$$
\sigma_{11}(\varphi=0)=\frac{E}{1+v}\left(\frac{\partial \varepsilon_{\psi}}{\partial \sin ^{2} \psi}\right),
$$

which is the well known $\sin ^{2} \psi$ equation. The bi-axial in-plane stress can then be determined from the slope of a $d$ versus $\sin ^{2} \psi$ plot. If the $d$ versus $\sin ^{2} \psi$ plot is linear, only two measurements at $\psi=0$ and $\psi$, the so-called 'two-tilt' method, are required, as expressed in equation (6) with $\varphi=0$ :

$$
\sigma_{11}(\varphi=0)=\frac{E}{1+v} \frac{1}{\sin ^{2} \psi} \frac{\cot \theta}{2}\left(2 \theta_{0}-2 \theta_{\psi}\right)=K \Delta 2 \theta,
$$

where the stress constant

$$
K=\frac{E}{1+v} \frac{1}{\sin ^{2} \psi} \frac{\cot \theta}{2} .
$$

$\theta$ is the Bragg angle, which is about $\left(\theta_{0}+\theta_{\psi}\right) / 2$, where $\theta_{0}$ and $\theta_{\psi}$ are the diffracting angles at $\psi=0$ and $\psi$, respectively. This two-tilt $\sin ^{2} \psi$ method using an area detector is very useful because the displacement error due to sample rotation is eliminated.

\subsection{The $\cos \alpha$ method}

The two-dimensional $\cos \alpha$ method was first proposed in the late 1970s (Taira et al., 1978; Taira \& Tanaka, 1979) using photographic film. It was applied to the image plate in the early 1990s (Yoshioka \& Ohya, 1992), and has been further developed for advanced use since the late 1990s (Sasaki et al., 1997; Sasaki \& Kobayashi, 2009; Sasaki et al., 2014). Suppose that a diffraction ring forms on the image plate through which the incident beam passes with an angle of $\psi_{0}$ and $\varphi_{0}$ from the $z$ and $x$ axes, as shown in Fig. 1. The strain $\varepsilon_{\alpha}$ projected along a direction with angle $\alpha$ can be expressed as the following, under the same assumptions as in the $\sin ^{2} \psi$ method:

$$
\begin{aligned}
\varepsilon_{\alpha}= & \sigma_{11}\left(\frac{1}{E}\right)\left[n_{1}^{2}-v\left(n_{2}^{2}+n_{3}^{2}\right)\right]+\sigma_{22}\left(\frac{1}{E}\right)\left[n_{2}^{2}-v\left(n_{1}^{2}+n_{3}^{2}\right)\right] \\
& +\sigma_{12}\left[\frac{2(1+v)}{E}\right]\left(n_{1} n_{2}\right),
\end{aligned}
$$

where $n_{1}, n_{2}, n_{3}$ are the directional cosines of the normal of the diffracting plane with respect to the axes in the sample coordinates and depend on $\alpha, \theta, \psi_{0}$ and $\varphi_{0}$. Substituting $-\alpha, \pi+\alpha$ and $\pi-\alpha$ for $\alpha$ in equation (7), we can then obtain the following relations:

$$
\begin{aligned}
& a_{1}\left(\varphi_{0}\right)=\frac{1}{2}\left[\left(\varepsilon_{\alpha}-\varepsilon_{\pi+\alpha}\right)+\left(\varepsilon_{-\alpha}-\varepsilon_{\pi-\alpha}\right)\right], \\
& a_{2}\left(\varphi_{0}\right)=\frac{1}{2}\left[\left(\varepsilon_{\alpha}-\varepsilon_{\pi+\alpha}\right)-\left(\varepsilon_{-\alpha}-\varepsilon_{\pi-\alpha}\right)\right] .
\end{aligned}
$$

Expressing equations (8) and (9) in terms of stress after reexpressing the directional cosines leads to the final relationships for this method:

$$
\begin{gathered}
\sigma_{11}\left(\varphi_{0}=0\right)=-\frac{E}{(1+\nu)} \frac{1}{\sin 2 \eta} \frac{1}{\sin 2 \psi_{0}}\left(\frac{\partial a_{1}}{\partial \cos \alpha}\right), \\
\sigma_{12}\left(\varphi_{0}=0\right)=\frac{E}{2(1+\nu)} \frac{1}{\sin 2 \eta} \frac{1}{\sin \psi_{0}}\left(\frac{\partial a_{2}}{\partial \sin \alpha}\right),
\end{gathered}
$$

where $\eta$ is defined in Fig. 1. For a given $\psi_{0}$ and $\eta$, we can get the in-plane normal stress from the slope of the $a_{1}$ versus $\cos \alpha$ plot when $\varphi_{0}=0$ from equation (10).

There are other two-dimensional methods such as $\mathrm{XRD}^{2}$ or full-ring fitting. However, only $\cos \alpha$ is chosen in this study as a representative of two-dimensional methods because the fundamental equation of the $\mathrm{XRD}^{2}$ method is basically identical to equation (7), and it measures only part of the Debye ring (Miyazaki \& Sasaki, 2016), while the $\cos \alpha$ and full-ring methods use all of the information. We have also shown experimentally that, when using the same dataset, there was no difference between the $\cos \alpha$ and full-ring fitting methods (Ramirez-Rico et al., 2016).

In summary, equations (5) and (10) are the basic equations for the $\sin ^{2} \psi$ and $\cos \alpha$ analysis in isotropic, homogeneous and bi-axial stress conditions. For anisotropic, heterogeneous or multi-axial stress cases, these equations have to be modified as reported previously by Noyan \& Cohen (1987) and Dölle (1979) for the $\sin ^{2} \psi$ method and by Sasaki and co-workers (Sasaki et al., 2014; Sasaki \& Kobayashi, 2009) for the $\cos \alpha$ method. 


\section{Experiment and data analysis}

\subsection{Samples}

Three samples were tested: (1) $\mathrm{Fe}$ reference powders $(E: 224 \mathrm{GPa}, v$ : $0.28)$ provided by the manufacturer of the portable X-ray device, (2) solid bars of 1018 mild carbon steel (E: $203 \mathrm{GPa}, v: 0.28$ ) and (3) solid bars of 6061 aluminium alloy (E: $69 \mathrm{GPa}, v: 0.33$ ) purchased from the McMaster-Carr supply company. The $\mathrm{Fe}$ reference powders were annealed to remove any pre-existing defects and residual stress. With average grain size $>1 \mu \mathrm{m}$, size or strain broadening effects were not expected in the diffraction patterns of these powders. Measurements of the reference powders were therefore used to characterize the intrinsic instrument precision of the portable X-ray device. The solid bar samples of $\mathrm{Fe}$ and $\mathrm{Al}$, both with dimensions $0.25 \times 0.25 \times 24^{\prime \prime}\left(1^{\prime \prime}=25.4 \mathrm{~mm}\right)$, were loaded in tension for accuracy testing. Since the Fe is the main testing sample, we measured the microstructure of the carbon steel bar by optical microscopy (ZEISS Axio Scope) and electron backscatter diffraction (EBSD) (JEOL 5600 equipped with HKL Nordlys and Channel 5 software; Oxford Instruments, Abingdon, Oxfordshire, UK), as shown in Fig. 2. These measurements clearly display elongated grains along the vertical $(\mathbf{Z})$ direction. X-ray diffraction from 20 to $110^{\circ}$ in $2 \theta$ with a $\mathrm{Cu}$ source (D500 diffractometer) probing both the top cross section ( $X Y$ plane) and the side wall ( $X Z$ plane) revealed that some (110) texture was developed in the $\mathbf{Z}$ direction, similar to wire extrusion, while grains parallel to the side wall ( $X Z$ plane) are distributed quite isotropically. The 110 peak intensity ratio $\left[I_{110} /\left(I_{110}+I_{200}+I_{211}+I_{220}\right)\right]$ is 0.74 , 0.66 and 0.63 for the top cross section, side wall and JCPDS reference, respectively. The EBSD analysis from the top cross section also shows a larger population of (110) grains than seen in the side walls.

\subsection{In situ diffraction measurements in tensile loading}

The 1018 mild carbon steel and $6061 \mathrm{Al}$ alloy bar samples were placed in an Instron 5984 Universal Testing Machine for tensile loading up to 300 and $200 \mathrm{MPa}$, respectively. Experiments were carried out under load control to keep the elastic stress constant during diffraction measurements, and samples were held by pneumatic grips as shown in Fig. 3. A miniature portable X-ray apparatus was mounted next to the sample (sample-to-detector distance $30-60 \mathrm{~mm}$ ) with various tilt angles $\left(\psi_{0}\right.$ in the range $5-48^{\circ}$ ). The load was increased in 25 or $50 \mathrm{MPa}$ steps. At each load we performed diffraction measurements for about $90 \mathrm{~s}$ for $\mathrm{Fe}$ and $120 \mathrm{~s}$ for Al. The specifications of the portable X-ray unit are the following: tube voltage $(30 \mathrm{kV})$, current $(1 \mathrm{~mA})$, source $(\mathrm{Cr} K \alpha$ with $\beta$ filter), X-ray energy $(\lambda=2.29 \AA, E=5.4 \mathrm{keV})$, collimator
( $1 \mathrm{~mm}$ diameter), beam size ( $2 \mathrm{~mm}$ diameter), sensor unit weight $(5 \mathrm{~kg})$, power supply weight $(6 \mathrm{~kg})$, energy consumption ( $80 \mathrm{~W}$ in operation, $30 \mathrm{~W}$ in standby mode). The specific model and company information about this portable device is not disclosed here for objective evaluation.

\subsection{Measurement parameters}

Instrumental parameters which influence the precision and accuracy of the stress measurement include beam size and divergence, use of a $K \beta$ filter, sample-to-detector distance, choice of tilt angle $\psi_{0}$, detector resolution, peak fitting

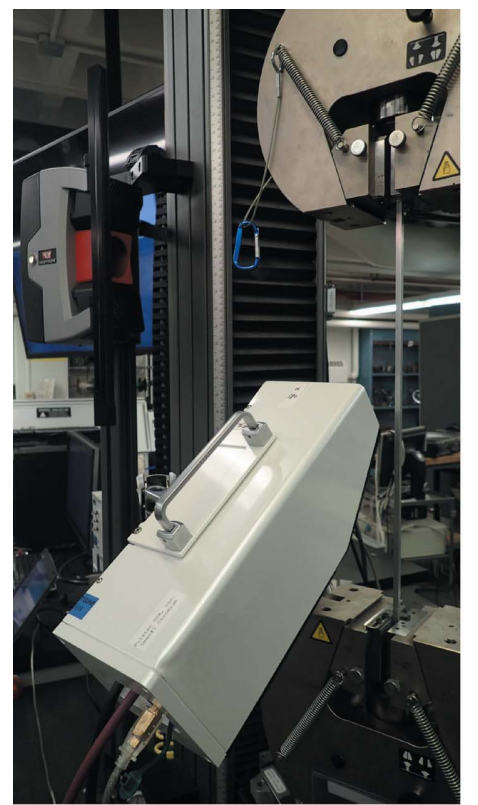

(a)

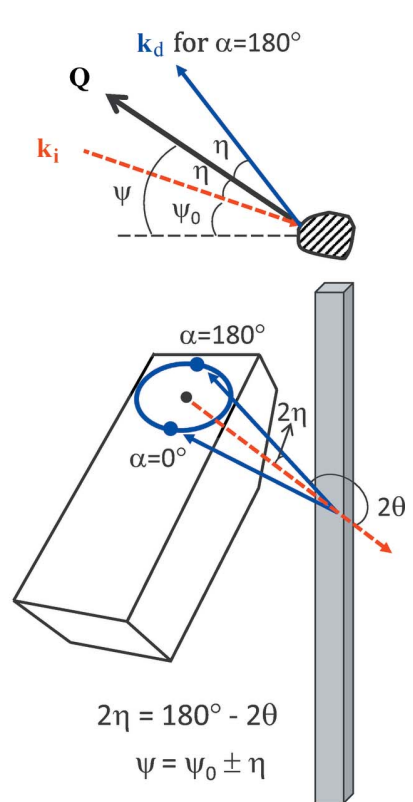

(b)
Figure 3

(a) Experimental setup for the in situ tensile loading test with a $24^{\prime \prime}$ long rectangular bar. (b) Schematic illustration with diffraction geometry. The portable device was tilted to probe various diffraction vectors. Note that $2 \eta=2 \pi-2 \theta, \psi=\psi_{0} \pm \eta$. 


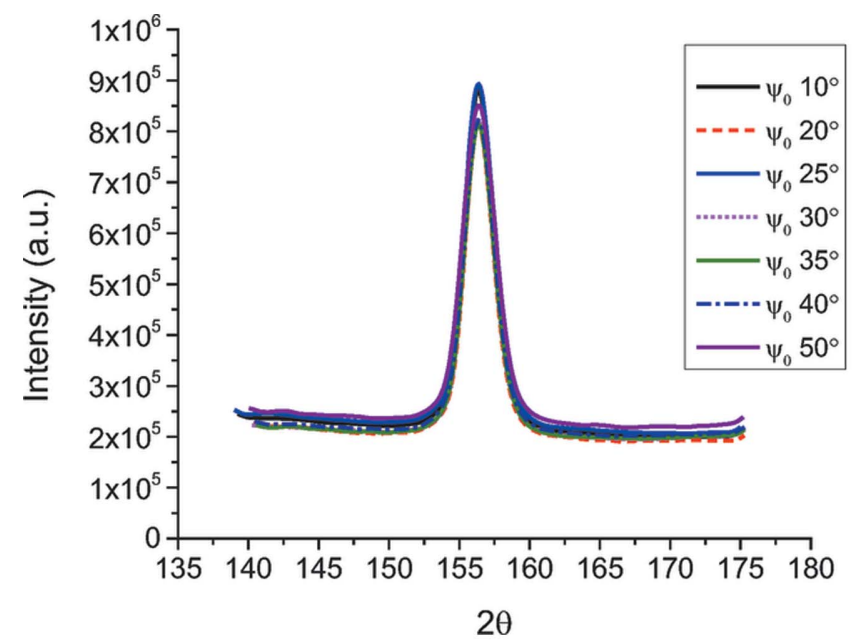

(a)

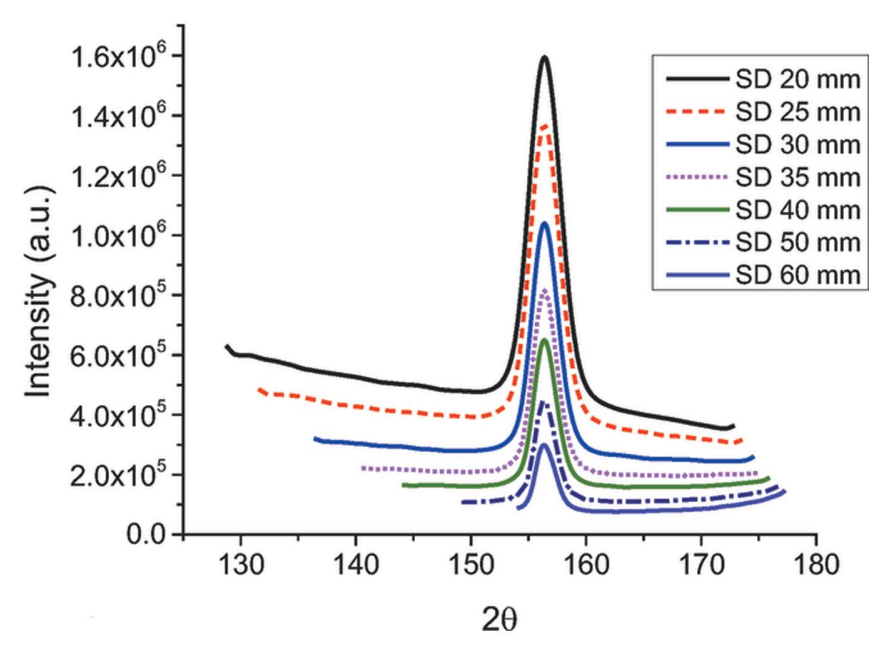

(b)

Figure 4

Fe 211 powder diffraction patterns obtained at $\alpha=0$, with various $(a) \psi_{0}$ at $\mathrm{SD}=35 \mathrm{~mm}$ and $(b) \mathrm{SD}$ at $\psi_{0}=35^{\circ}$. The intensity and $2 \theta$ range are strongly dependent on SD.

function, scanning time etc. Among these parameters, the most important are sample tilt angle $\left(\psi_{0}\right)$ and sample-to-detector distance (abbreviated as 'SD' hereafter).

Fig. 4 shows the one-dimensional diffraction profiles integrated from the Debye rings of Fe reference powders. If the displacement error is autocorrected by SD adjustment so that the peak centers are shifted to the position expected from Bragg's law, there is no noticeable difference due to sample tilt angle $\left(\psi_{0}\right)$, as shown in Fig. 4(a). However, the $2 \theta$ range and intensity are directly influenced by the SD. The use of large SDs results in a narrower $2 \theta$ range and thus in higher resolution, but at the cost of sacrificing intensity. Meanwhile, as the SD becomes smaller, the peak intensity increases with increasing $2 \theta$ coverage. For example, for a given detector configuration $(29.7 \mathrm{~mm}$ outer radius with $3 \mathrm{~mm}$ inner radius,

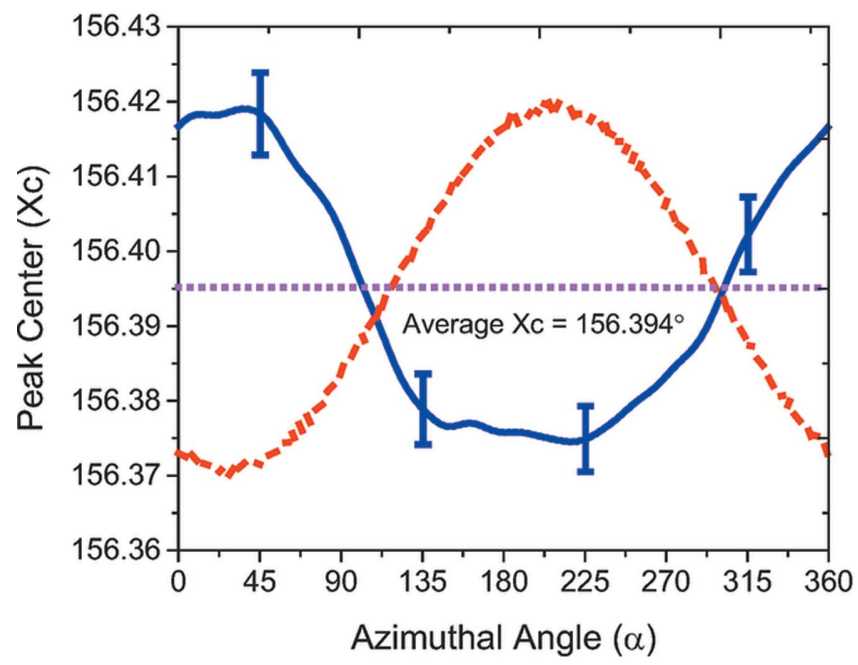

(a)
$50 \mu \mathrm{m}$ pitch, 534 radial pixels), a $60 \mathrm{~mm} \mathrm{SD} \mathrm{covers} 23^{\circ}$ in $2 \theta$ resulting in $0.04^{\circ}$ resolution, whereas coverage doubles when the $\mathrm{SD}$ is reduced to $20 \mathrm{~mm}$ at the cost of increasing the resolution to $0.08^{\circ}$. In general, there is a trade-off between intensity and resolution in the chosen sample-to-detector distance. This effect is somewhat mitigated because, as seen in Fig. 4(b), the peak-to-background ratio remains about the same. Thus it is better to keep the SD large as long as the reflection peak of interest falls within the range covered by the image plate.

\subsection{Instrument resolution and beam center calibration}

Before addressing questions of precision and accuracy under field measurement conditions, the intrinsic instrument

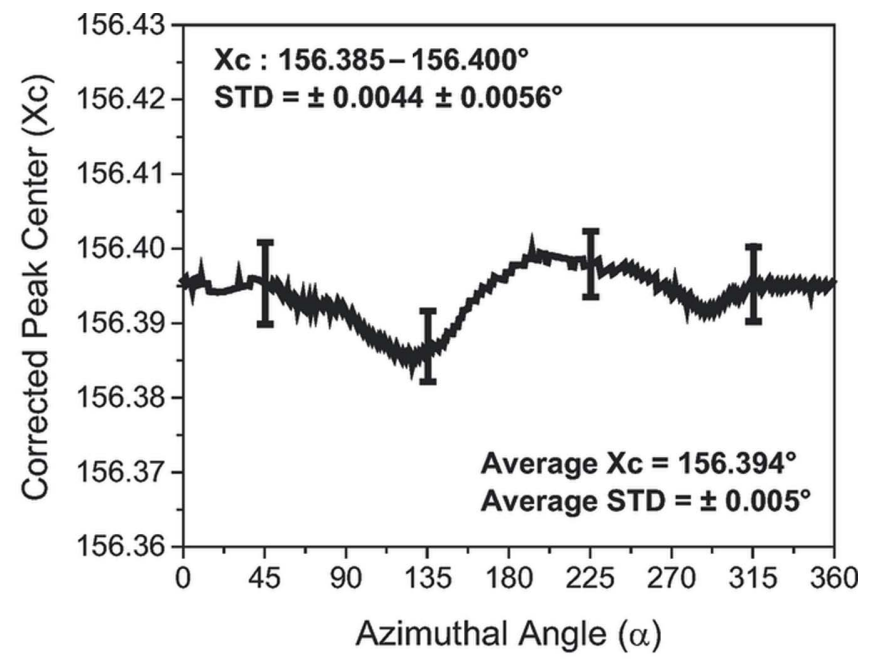

(b)

Figure 5

(a) Peak center position versus $\alpha$ angle (blue solid line) and calibration curve (red dashed line) resulting from the $25 \mu \mathrm{m}$ beam center offset. (b) Calibrated peak center plot obtained by adding the two curves from $(a)$. This corrected profile is used for the stress calculations expressed in equation (10). 
Table 1

Measured stress from the $\sin ^{2} \psi$ or $\cos \alpha$ method from different data types.

The error $( \pm 2 \mathrm{MPa})$ is the standard deviation of the five measurements. Scans were done at $35^{\circ} \psi_{0}$ and $38 \mathrm{~mm} \mathrm{SD}$.

\begin{tabular}{lrr}
\hline Data type & \multicolumn{1}{c}{$\sin ^{2} \psi$} & \multicolumn{1}{c}{$\cos \alpha$} \\
\hline Non-calibrated data (Fig. 5a, blue solid line) & $-2 \pm 2 \mathrm{MPa}$ & $35 \pm 2 \mathrm{MPa}$ \\
Calibrated data (Fig. 5b, black solid line) & $0 \pm 2 \mathrm{MPa}$ & $0 \pm 2 \mathrm{MPa}$ \\
\hline
\end{tabular}

resolution, i.e. the best attainable precision in laboratory conditions, and raw data calibration procedure must be established.

The spatial resolution of each detector pixel is determined by the pitch of the laser scan, which can be adjusted from 20 to $100 \mu \mathrm{m}$; a default value of $50 \mu \mathrm{m}$ was used throughout all the measurements in this study. For a given detector size $(59.4 \mathrm{~mm}$ diameter with $6 \mathrm{~mm}$ diameter hole in the center for beam penetration and collimator installation), $50 \mu \mathrm{m}$ radial pitch and $0.72^{\circ}$ angular step in a spiral reading generates 534 data points in $2 \theta$ and 500 data values at each $2 \theta$ in terms of the $\alpha$ angle.

We performed preliminary tests with annealed Fe reference powders at one of the recommended settings: $35^{\circ} \psi_{0}, 38 \mathrm{~mm}$ $\mathrm{SD}$. A total of 80 measurements were repeated over the course of $48 \mathrm{~h}$ with multiple cycles, turning the apparatus on/off, but without moving any part of the sample or machine for the best true instrument precision. In order to quantify every source of uncertainty, all data analyses were done fully manually in the following steps: (1) Raw intensity versus $2 \theta$ data were exported for $500 \alpha$ angles. (2) Peak center positions in $2 \theta$ were found via pseudo-Voigt peak fitting for 40000 datasets (80 scans $\times 500$ angles). (3) Residual stresses were calculated via the $\sin ^{2} \psi$ and $\cos \alpha$ methods for five selected datasets, both with and without beam center correction using equations (5) and (10), and then compared with $\cos \alpha$ stress values obtained from the instrument's own analysis software.

Fig. 5(a) shows the averaged peak center from 80 measurements versus azimuthal angle, $\alpha$, as a solid blue line. Error bars ( \pm one standard deviation, about $0.005^{\circ}$ ) are displayed only in a few places, but they were fairly consistent for all $\alpha$ angles. The blue curve with $0.025^{\circ}$ amplitude in $2 \theta$ indicates that the beam center is offset by $25 \mu \mathrm{m}$ from the exact center point, which is half of the pixel resolution. Owing to the difficulty of the X-ray beam alignment and the offset being less than the pitch resolution, this device has the internal calibration curve shown as a red dashed line in Fig. 5(a). Adding those two curves gives the corrected final peak positions shown in Fig. 5(b). Since these calibration curves change according to the $\mathrm{SD}$, the measurement error in sample height $(\Delta \mathrm{SD})$ introduces error in the stress measurement. The typical way to calculate the SD is to spread a mixture of reference Fe powders in vacuum grease on the sample and let the machine calculate the SD from the Fe 211 peak. Afterwards, the grease is wiped off, exposing the sample surface for measurement. Since the wavelength and Fe 211 Bragg angle are known, the accurate SD can be calculated and users can employ this value for their actual samples. The precision in SD measurement is
Table 2

List of uncertainty types and values.

Measurement precision can be represented by the Type 3 error, which is about $2 \mathrm{MPa}$ in the case of Fe. All stress values calculated in $\$ 3.4$ are based on the reference powder modulus, $E=224 \mathrm{GPa}$.

\begin{tabular}{|c|c|c|c|c|}
\hline Type & Uncertainty & $\begin{array}{l}\Delta 2 \theta \\
\left(^{\circ}\right)\end{array}$ & $\begin{array}{l}\Delta d / d \\
(\mu \varepsilon)\end{array}$ & $\begin{array}{l}\sigma \\
(\mathrm{MPa})\end{array}$ \\
\hline 1 & Diffraction peak fitting error $(\Delta 2 \theta$, Fig. 4$)$ & 0.003 & 5 & 1 \\
\hline 2 & $\begin{array}{l}\text { Average standard deviation from all } \alpha \text { angles } \\
\text { (Fig. } 5 b \text { ) }\end{array}$ & 0.005 & 9 & 2 \\
\hline 3 & $\cos \alpha$ fitting error [equation (10)] & NA & NA & 2 \\
\hline 4 & $\sin ^{2} \psi$ fitting error [equation (5)] & NA & NA & 4 \\
\hline 5 & Maximum deviation among $\alpha$ angles (Fig. $5 b$ ) & 0.015 & 28 & 6 \\
\hline 6 & Detector spatial resolution $(50 \mu \mathrm{m})$ & 0.05 & 90 & 20 \\
\hline 7 & FWHM of the peak (Fig. 4) & 2.3 & $\sim 4000$ & NA \\
\hline
\end{tabular}

less than $10 \mu \mathrm{m}$, so the stress error associated with SD uncertainty is negligible.

During the $50 \mathrm{~s}$ period of data reading and analysis after the $40 \mathrm{~s}$ beam exposure, the portable machine goes through fitting and calibration processes, and then provides an in-plane residual stress of $0 \pm 2 \mathrm{MPa}$, which is identical to that provided by our manual $\cos \alpha$ analysis. However, if the calibration step is missed, i.e. the blue line in Fig. 5(a) is used, the stress from the $\cos \alpha$ method is $35 \pm 2 \mathrm{MPa}$. In the $\sin ^{2} \psi$ method, ${ }^{3}$ the measured stresses are $-2 \pm 2 \mathrm{MPa}$ for noncalibrated data (Fig. $5 a$, blue) and $0 \pm 2 \mathrm{MPa}$ for the calibrated data (Fig. 5b), which proves that the $\sin ^{2} \psi$ method is not sensitive to the calibration process because it probes $\psi$-dependent strain at a fixed $\alpha$, while the $\cos \alpha$ method uses $\alpha$-dependent strain at a fixed $\psi$ (Table 1$)$. However, the $\sin ^{2} \psi$ method requires samples to be at the exact center of rotation for an accurate measurement, which is challenging and time consuming for such a small portable X-ray device. One can use the 'two-tilt' $\sin ^{2} \psi$ method to avoid the sample rotation issue by using only two points at $\alpha=0$ and $180^{\circ}$ from the Debye ring, but its accuracy is also sensitive to the calibration process, just as in the $\cos \alpha$ method, when non-calibrated data are used.

Uncertainty arises at each analysis step and possible error sources are summarized in Table 2 . The overall uncertainty is a combination of the contributions related to (1) diffraction peak fitting error (Type 1), (2) the repeatability of the peak center calculation (Type 2), and (3) linear fitting of equation (10) or (5) for stress determination in the $\cos \alpha$ (Type 3) or $\sin ^{2} \psi$ (Type 4) methods, respectively. As shown in Table 1, without sample heterogeneity or displacement errors, the overall stress error can be as good as $2 \mathrm{MPa}$ for ferritic steel. Types 5-7 in Table 2 are listed as bounds of maximum errors. Note that these uncertainties are measured at a fixed $\psi_{0}$ and $\mathrm{SD}$, and the precision was obtained at the best static laboratory conditions, but those parameters are not often achievable in field measurements. Therefore, the effects of $\psi_{0}$ and SD on the precision and accuracy need to be measured for practical applications.

\footnotetext{
${ }^{3}$ For the $\sin ^{2} \psi$ method, six $\psi_{0}$ counterclockwise rotations $\left(0,5,10,15,20,25^{\circ}\right)$ were made in laboratory coordinates, which corresponds to the six $\psi$ angles $\left(11.8,16.8,21.8,26.8,31.8,36.8^{\circ}\right)$ in sample coordinates at $\alpha=180^{\circ}$.
} 


\section{Results}

\subsection{Precision test with ferrite reference powders}

The dependence of the precision on the choice of measurement parameters ( $\psi_{0}$ and SD) was investigated using annealed ferrite reference powders. The sample tilt $\left(\psi_{0}\right)$ angle varied from 5 to $60^{\circ}$ for each sample-to-detector distance (SD), which itself ranged from 20 to $60 \mathrm{~mm}$. For the statistical analysis, this whole process was repeated five times (for a total of 540 measurements) at different sample positions. Since equivalency between $\cos \alpha$ and $\sin ^{2} \psi$ was shown in $\S 3.4$ and by other authors (Ling \& Lee, 2015; Ramirez-Rico et al., 2016; Miyazaki \& Sasaki, 2016) under a homogeneous bi-axial stress state, we used stress values calculated with the instrument software by the $\cos \alpha$ method. Reference powders were expected to exhibit no stress irrespective of the chosen value for the aforementioned parameters.

The averaged stress values versus tilt angles $\left(\psi_{0}\right)$ are plotted in Fig. 6, showing that there is a proper range of sample tilt angle for precise and accurate measurements: $20 \leq \psi_{0} \leq 50^{\circ}$. Outside that range, measurements become imprecise and inaccurate. At lower tilt angles, $\psi_{0} \leq 15^{\circ}$, the measured stresses strongly depend on $\mathrm{SD}$, while monotonic decreases are observed at $\psi_{0} \geq 55^{\circ}$. This is consistent with our previous simulation results (Ramirez-Rico et al., 2016) in that orientation error, $\Delta \psi_{0}$, is more significant at lower $\psi_{0}$, and that beam defocusing becomes important at higher $\psi_{0}$. The inset of Fig. 6 shows that for this particular apparatus an SD of $35 \mathrm{~mm}$ results in good accuracy regardless of tilt angle. The same data as in Fig. 6 are plotted in Fig. 7, as a function of SD for given $\psi_{0}$ angles. The calculated stress tends to increase with increasing SD, and its effect becomes large at lower tilt angles.

Fig. 8 displays one standard deviation from five measurements at each measurement condition ( $\psi_{0}$ and SD) as a representative parameter quantifying precision. At intermediate ranges $\left(20 \leq \psi_{0} \leq 50^{\circ}, 20 \leq \mathrm{SD} \leq 60 \mathrm{~mm}\right)$, the mean stress is $3 \mathrm{MPa}$; the precision is about $4 \mathrm{MPa}$ for five different spots and $3 \mathrm{MPa}$ for five continuous scans at one spot. Fig. 8 also illustrates that the sample-to-detector distance is not a critical parameter unless $\psi_{0}$ is too low or high. More errors at very low tilt angles are inevitable because below $\psi_{0}=11.8^{\circ 4}$ we lose the independent information volume that the Debye ring captures for $\cos \alpha$ stress calculation. The wide beam spread at high $\psi_{0}$ angle also causes errors in both precision and accuracy. In general, these undesirable measurement conditions should be avoided, although in field conditions this might not be possible. For such cases, it is necessary to know the uncertainty budgets caused by measurement parameters as shown in Figs. 6, 7 and 8.

\subsection{Accuracy test with solid samples under in situ tensile loading}

Obtaining the true stress state is a challenging task for both diffraction-based techniques and destructive mechanical

\footnotetext{
${ }^{4} \psi=\psi_{0} \pm \eta, \eta=11.8^{\circ}$ for Fe 211 , so the $\psi$ information becomes redundant at $\psi_{0} \leq 11.8^{\circ}$.
}

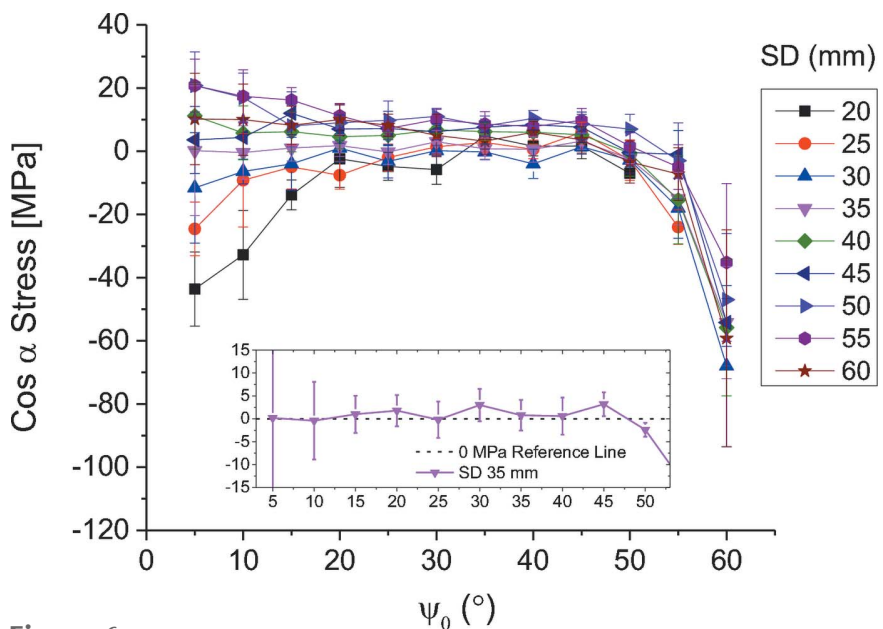

Figure 6

Measured stress values versus $\psi_{0}$ angles. Measured stresses deviate from zero considerably at $\psi_{0} \leq 15^{\circ}$ and $\psi_{0} \geq 55^{\circ}$.

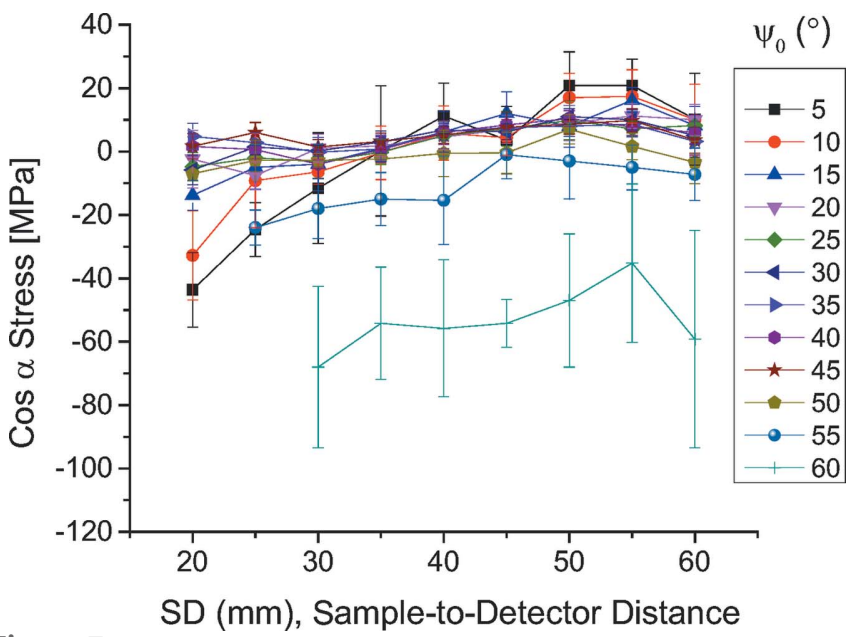

Figure 7

Measured stress values versus SD. The SD effect is minor around intermediate tilt angles $\left(20 \leq \psi_{0} \leq 50^{\circ}\right)$, but becomes significant at lower $\psi_{0}$.

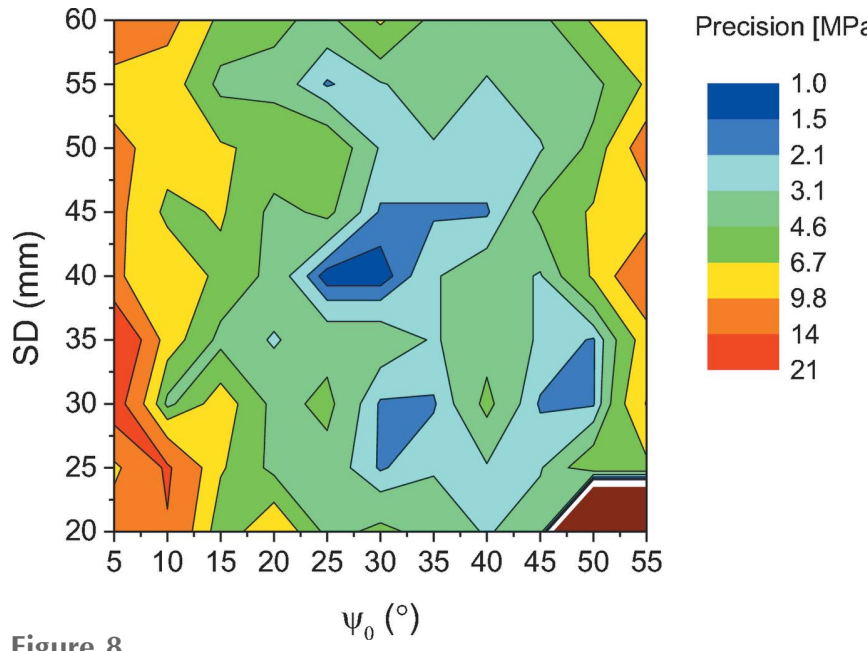

Figure 8

Contour plot of standard deviation (precision) out of five measurements at each $\psi_{0}$ and SD. The precision error is less sensitive to SD around intermediate tilt angles $\left(20 \leq \psi_{0} \leq 50^{\circ}\right)$, while $\psi_{0}$ shows a wider variation in all SD ranges. 
methods; thus they are often used together as complementary tools. The best way to evaluate measurement accuracy is to compare measurements with known applied stresses. Two rectangular solid bars (1018 steel and 6061 aluminium) were loaded under tensile stress using the apparatus and methodology described in $\$ \S 3.1$ and 3.2; a tensile stress was applied at several tilt angles $\left(\psi_{0}=12,25,35\right.$ and $\left.45^{\circ}\right)$ in 50 or $25 \mathrm{MPa}$ steps. Fig. 9 shows raw two-dimensional diffraction profiles and the Debye ring distortion from each sample at various $\psi_{0}$. The carbon steel shows no preferred orientation, at least in the 211 reflections within the diffracting volume, and the peak center is very stable throughout all $\alpha$ angles. However, the
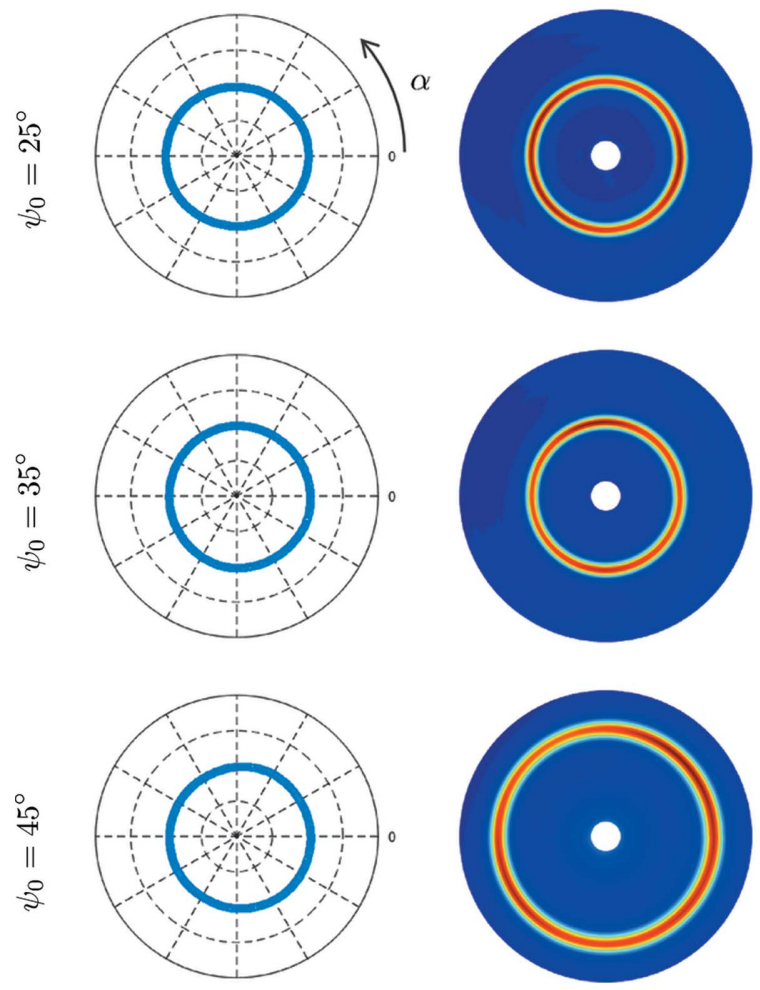

(a)
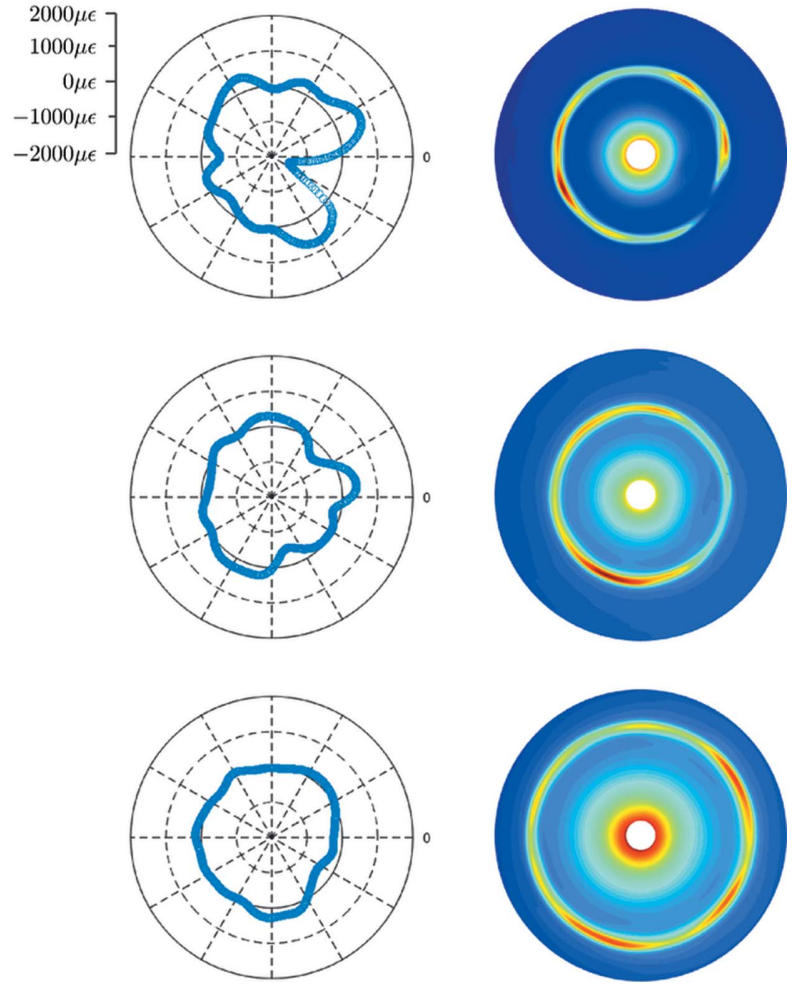

$(b)$

Figure 9

Normalized two-dimensional diffraction patterns of $(a)$ mild carbon steel 1018 and $(b)$ aluminium 6061 alloy at zero applied stress. The Debye ring moves outside as $\psi_{0}$ increases owing to the corresponding SD increase. The carbon steel shows a homogeneous grain distribution, while the aluminium alloy is textured. The left column displays how much the Debye ring is distorted by the peak center positions (polar scale from -2000 to $2000 \mu \varepsilon$ ). The sampleto-detector distances are $34,34,50 \mathrm{~mm}$ for steel and $41,41,52 \mathrm{~mm}$ for aluminium.

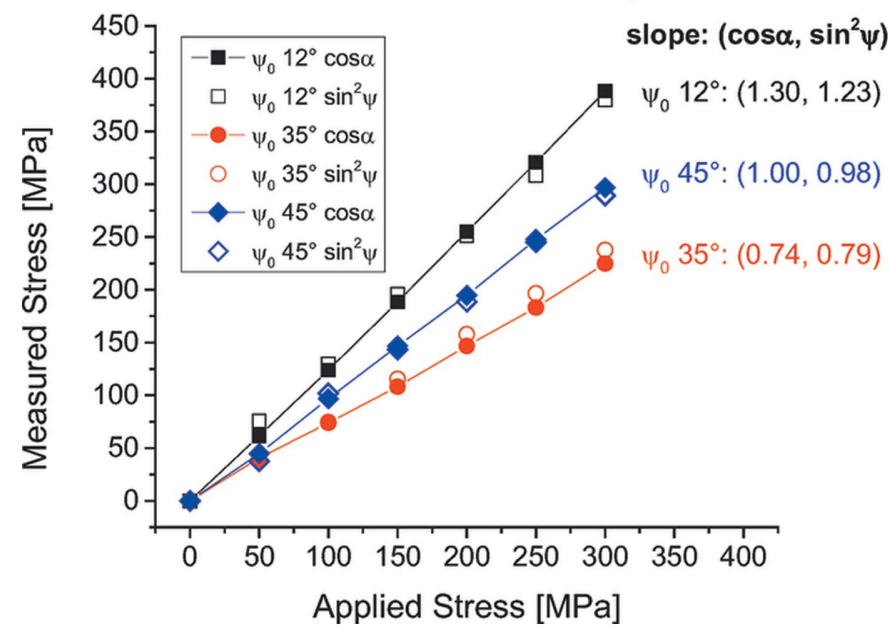

Figure 10

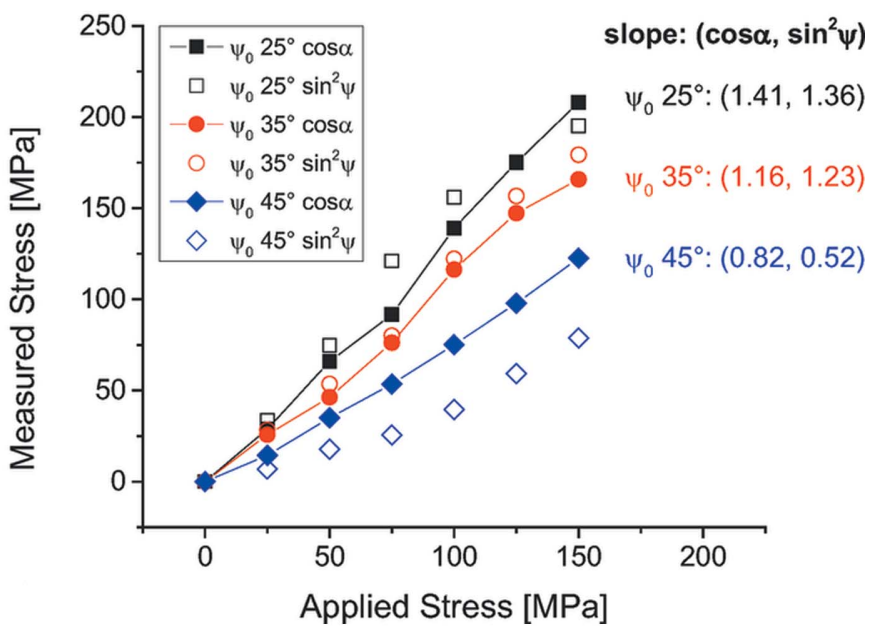

(b)

Measured versus applied stress from (a) the 1018 carbon steel 211 peak and $(b)$ the 6061 aluminium alloy 222 peak. For steel, both $\sin ^{2} \psi$ and $\cos \alpha$ show identical results for a given $\psi_{0}$, while aluminium does not show reliable results. Initial residual stresses were offset to zero. The steel data at $\psi_{0}=12^{\circ}$ are plotted instead of at $\psi_{0}=25^{\circ}$, because the data at $25^{\circ}$ are nearly the same as those at $35^{\circ}$. 
Table 3

Applied and measured $\cos \alpha$ stresses (MPa) with carbon steel at each tilt angle $\left(\psi_{0}\right)$.

Average values and error bars were obtained from five repeated measurements. Note that average values (accuracy) can be different if the internal calibration profile changes, while error bars (precision) are more sensitive to measurement conditions. For the accuracy evaluation, the measured/applied stress ratio, as shown in Table 4, is the better way because the true residual stress state at zero load is unknown. The best precision is achieved at $\psi_{0}=35^{\circ}$.

\begin{tabular}{|c|c|c|c|c|c|c|c|c|c|c|}
\hline \multirow[b]{2}{*}{ Applied stress (MPa) } & \multicolumn{10}{|c|}{ Measured stress (MPa) at each tilt angle $\left(\psi_{0}\right)$} \\
\hline & $5^{\circ}$ & $10^{\circ}$ & $15^{\circ}$ & $20^{\circ}$ & $25^{\circ}$ & $30^{\circ}$ & $35^{\circ}$ & $40^{\circ}$ & $45^{\circ}$ & $48^{\circ}$ \\
\hline 0 & $67(9)$ & $30(11)$ & $27(12)$ & $25(9)$ & $20(10)$ & $1(13)$ & $-19(8)$ & $-50(15)$ & $-98(26)$ & $-138(22)$ \\
\hline 300 & $558(68)$ & $463(28)$ & $372(7)$ & $311(9)$ & $254(30)$ & $205(9)$ & $198(6)$ & $208(17)$ & $198(22)$ & $166(23)$ \\
\hline
\end{tabular}

aluminium 222 intensity is weak and inhomogeneous. The Debye ring is also distorted as peak centers fluctuate around the ring. Therefore, steel samples are expected to show more reliable results than aluminium. Also note that the SD was rather large (about $50 \mathrm{~mm}$ ) for $\psi_{0}=45^{\circ}$, because the minimum sample-to-detector distance increases with increasing tilt angle owing to the fact that the sample is very long and interferes with the instrument at high tilt angle (see Fig. 3).

Measured stresses from both the $\cos \alpha$ and the two-tilt $\sin ^{2} \psi$ methods are plotted against applied stress in Fig. 10. As a measure of accuracy, the slope (ratio of measured over applied stress) was calculated for each set of tilt angle conditions and noted in Fig. 10. The steel sample shows that both analysis methods yield similar results and there is a clear $\psi_{0}$ dependency in terms of accuracy. For example, measurement was most accurate and precise at $\psi_{0}=45^{\circ}$. As for the aluminium sample, $\psi_{0}$ dependency can be inferred, but $\cos \alpha$ and $\sin ^{2} \psi$ generate quite different results, neither of which is accurate or precise for the tested angles.

Knowing that the accuracy depends on the tilt angle and that the steel sample is much more reliable than the aluminium sample, a series of new in situ tensile loading experiments $(0,100,200,300 \mathrm{MPa})$ were performed with a $24^{\prime \prime}$ long steel bar sample for a $\psi_{0}$-dependent accuracy test. A total of

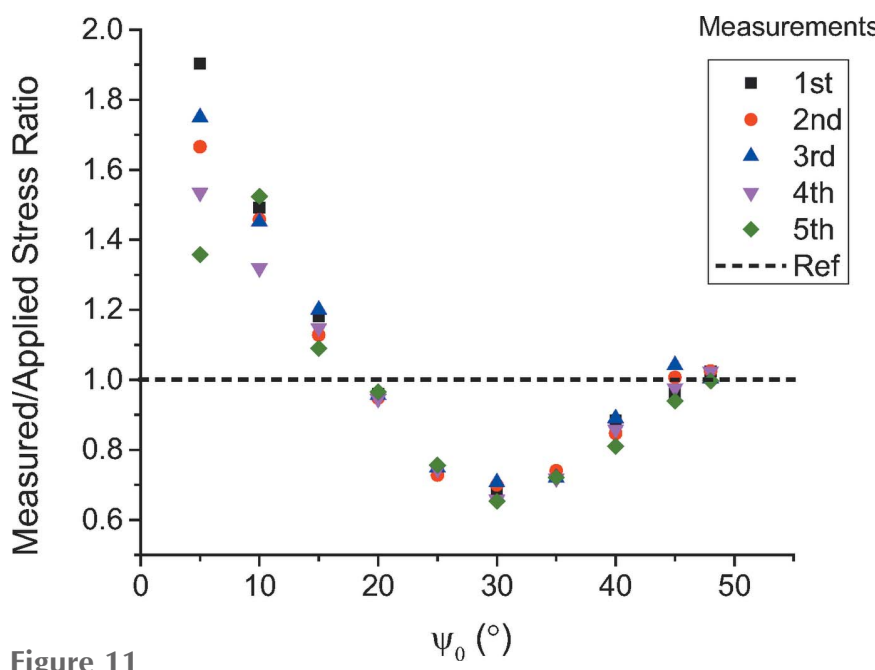

Figure 11

$\psi_{0}$ angle effect on the ratio of measured stress $(\cos \alpha)$ over applied stress for the 1018 carbon steel sample. A ratio of 1 indicates accurate stress measurement. Each ratio (slope) was determined from the four tensile loading steps $(0,100,200,300 \mathrm{MPa})$ and is summarized in Table 4. ten $\psi_{0}$ angles were tested from 5 to $48^{\circ} \psi_{0}$ with $5^{\circ}$ intervals as $\psi_{0}=0^{\circ}$ does not give a $\cos \alpha$ stress and $48^{\circ}$ is the maximum tilt angle to capture the 211 peak for a given sample geometry. This set of measurements was repeated five times (4 steps $\times$ $10 \psi_{0} \times 5=200$ measurements). Since the two-tilt $\sin ^{2} \psi$ results shown in Fig. 10(a) are very similar to the $\cos \alpha$ results as expected, $\cos \alpha$ values reported by the apparatus software were used throughout.

The ratios of measured over applied stress are plotted in Fig. 11 for all five measurements and the average stresses are listed in Table 3 along with standard deviations. As in the previous precision analysis, the measurements are quite repeatable at $20 \leq \psi_{0} \leq 48^{\circ}$, but the accuracy changes from 70 to $100 \%$ of the actual stress values. In other words, the conditions for the best precision $\left(\psi_{0}=35^{\circ}\right.$, with both $\mathrm{Fe}$ powders and the solid bar) and accuracy $\left(\psi_{0}=19\right.$ or $\left.45^{\circ}\right)$ are different. This result does not mean that the machine calibration is perfect at $45^{\circ}$ or offset by $30 \%$ at $35^{\circ}$ because there may be other factors affecting the accuracy, which are discussed in the next section. The measured/applied stress ratios at each value of $\psi_{0}$ are summarized in Table 4.

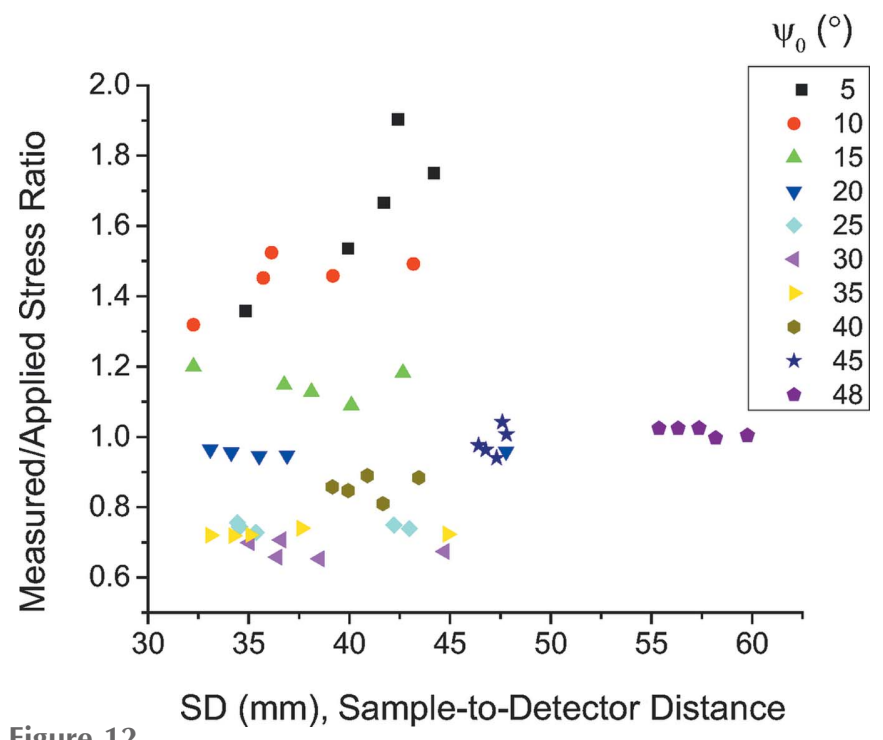

Figure 12

SD effect on the ratio of measured stress $(\cos \alpha)$ versus applied stress for the 1018 carbon steel sample. A ratio of 1 indicates accurate stress measurement. The accuracy of stress measurements is not sensitive to the SD except for the low $\psi_{0}$ angle regions where the measured stress increases with increasing SD. 
Table 4

Measured/applied stress ratios and fitting errors with carbon steel for the set of $\psi_{0}$ angles.

The $\cos \alpha$ stresses are based on Young's modulus of $203 \mathrm{GPa}$, which is measured from our own tensile testing.

\begin{tabular}{lllllllllll}
\hline$\psi_{0}$ & $5^{\circ}$ & $10^{\circ}$ & $15^{\circ}$ & $20^{\circ}$ & $25^{\circ}$ & $30^{\circ}$ & $35^{\circ}$ & $40^{\circ}$ & $45^{\circ}$ & $48^{\circ}$ \\
\hline Measured/applied & $1.64(0.21)$ & $1.45(0.08)$ & $1.15(0.04)$ & $0.95(0.01)$ & $0.77(0.07)$ & $0.68(0.02)$ & $0.72(0.01)$ & $0.86(0.03)$ & $0.99(0.04)$ & $1.01(0.01)$ \\
\hline
\end{tabular}

In order to check any SD effect, data from Fig. 11 are shown in Fig. 12 as a function of SD for each tilt angle. Fig. 12 indicates that the accuracy is very sensitive to the tilt angle $\left(\psi_{0}\right)$, but not to the SD, except at very low angles. Such low $\psi_{0}$ angle values are not pertinent in general because, as we have previously shown, they should be avoided.

The errors in Table 3 are much larger than the precisions obtained by testing the reference powder because of sampling statistics: the beam spot moves to another heterogeneous region whenever the tilt angle $\left(\psi_{0}\right)$ changes. For example, for the long sample, the beam spots move by 10 and $30 \mathrm{~mm}$ when the tilt angle changes from the default setting $\left(35^{\circ}\right)$ to a higher angle $\left(45\right.$ and $\left.55^{\circ}\right)$. This also makes the circular beam shape ( $2 \mathrm{~mm}$ diameter at $35^{\circ}$ ) elongated by 15 and $45 \%$ in the major axis $\left(2 \times 2.3 \mathrm{~mm}\right.$ at $45^{\circ}$ and $2 \times 2.9 \mathrm{~mm}$ at $55^{\circ}$, respectively). However, if the sample is small enough to fit under the detector (Fig. 1a), the beam center can be maintained on the original spot via sample translation. In such cases, the sample heterogeneity effect due to tilting is not significant. Thus, the long steel solid bar is not a good sample for the instrument precision test, just as the reference powder is not suitable for accuracy quantification.

\section{3. $h k l$ dependency on stress measurement}

In addition to the measurement parameters, the choice of diffracting planes $(h \mathrm{kl})$ and texture also affect stress precision and accuracy. Often, few choices are available for $h k l$ because

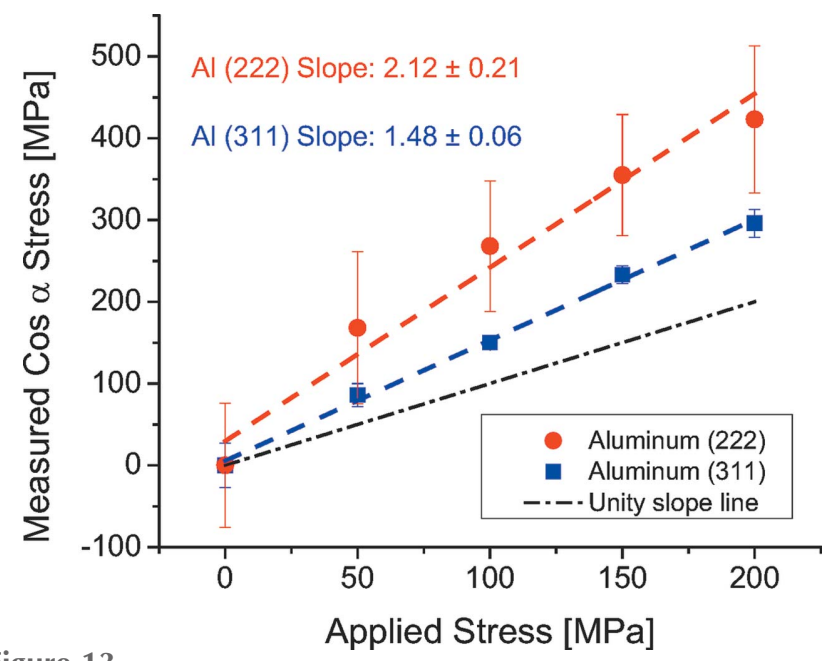

Figure 13

Measured versus applied stress from 6061 aluminium alloy 222 and 311 peaks at $20^{\circ} \psi_{0}$ and $22 \mathrm{~mm} \mathrm{SD}$. The stresses of the 222 peaks are about $40 \%$ higher than those of the 311 peaks. The 311 peak provides more reliable stress measurements than 222 . the $Q$ space is limited in this kind of back-reflection portable device, but depending on the phases, multiple peaks can sometimes be captured with a single exposure if the sample is brought close enough to the detector. While only the 211 peak was visible for steel samples within an acceptable SD range, in the case of aluminium both the 222 and 311 peaks were measured simultaneously using an SD of $22 \mathrm{~mm}$. A lower tilt angle $\left(20^{\circ}\right)$ was chosen for the smaller SDs because the minimum SD increases with increasing $\psi_{0}$ in this kind of long sample geometry, as depicted in Fig. 3. With the Cr target and detector size used here, some face-centered cubic (f.c.c.) materials with large lattice parameters such as $\mathrm{Al}, \mathrm{Ag}, \mathrm{Au}$ etc. can be measured in this manner.

Fig. 13 illustrates $h k l$-dependent stress values from the aluminium 222 and 311 peaks. The 311 reflection provides a smaller error than the 222 reflection, probably owing to the higher intensity and more uniform texture of 311 . The measured/applied stress ratios are $2.1 \pm 0.2$ and $1.5 \pm 0.1$ for the 222 and 311 reflections, respectively, while the $\mathrm{Fe} 211$ reflection shows reasonably accurate results $(0.95 \pm 0.01$ in Fig. 11) at $20^{\circ} \psi_{0}$. The result for the $\mathrm{Al} 222$ peak is consistent with the increasing trend of the stress with decreasing $\psi_{0}$ found in Fig. 10(b), but the overall trend in $\psi_{0}$-dependent accuracy is quite different from that for Fe, as shown in Fig. 11. Stresses calculated using the (222) planes are about $40 \%$ higher than when using the 311 reflection, which is unexpected because the anisotropy ratio of $\mathrm{Al}$ is only 1.2. The correct selection of $h \mathrm{kl}$ reflections for accurate stress determination is not simple (Clausen, Leffers \& Lorentzen, 2003) and anisotropy correction is a crucial component in stress analysis, which is discussed in the $§ 5$.

\section{Discussion}

In X-ray diffraction techniques, information comes only from the discrete sets of grains satisfying Bragg's condition, and strains from those lattice planes are used to estimate a bulk macrostress state. In addition to this selective nature of diffraction methods, elastic anisotropy, deformation history, texture, pseudo-macrostress etc. all affect the uncertainties of the measured stress values (James \& Cohen, 1978b). Since all the equations derived above are based on the assumption that the material's elastic response is isotropic and homogeneous with bi-axial plane stress near the surface region, measured stresses using those equations have no guarantee of being precise or accurate. A comprehensive review of such problems and suggested solutions is given in the literature (James \& Cohen, 1978b; Dölle, 1979; Noyan \& Cohen, 1987) for the traditional diffractometer. 
Table 5

Effect of theoretical and experimental diffraction elastic constant from in situ neutron diffraction measurement of b.c.c. ferritic steel (Daymond \& Priesmeyer, 2002).

The modulus unit is in GPa and $E_{\text {macro }}=211 \mathrm{GPa}$ for the isotropic model.

\begin{tabular}{llcccc}
\hline B.c.c. ferrite reflections & 110 & 200 & 211 & 310 \\
\hline$E_{h k l}$ & Kroner model & 216 & 170 & 216 & 184 \\
& $\quad$ ND experiment (Daymond & 226 & 170 & 216 & 185 \\
$\quad$ \& Priesmeyer, 2002) & & & & \\
& & & & & \\
$\sigma_{\text {measured }} / \sigma_{\text {applied }}$ & Isotropic model $[(1+v) / E]$ & 0.94 & 1.24 & 0.98 & 1.14 \\
& Kroner Model $\left(S_{2} / 2\right)$ & 0.96 & 1.00 & 1.00 & 0.99 \\
\hline
\end{tabular}

In this section, each source of error in this particular portable device with a two-dimensional detector is discussed. Since bi-axial plane stress is a reasonable assumption when using a low-energy X-ray source (such as $\mathrm{Cr} K \alpha$ ), unless there is a steep stress gradient within $25 \mu^{5}$ or so (James, 1977), the sample-related uncertainty comes from elastic anisotropy and the inhomogeneity associated with texture and plastic deformation. Effects of sampling and intensity (counting) statistics are also important and partially demonstrated in the case of aluminium (Fig. 9 and Fig. 10), but they are out of the scope of this article. Recent studies on how they scale with decreasing grain sizes can be found in the literature (Öztürk et al., 2014, 2015).

\subsection{Uncertainty from elastic anisotropy}

At the macroscopic scale, the mechanical response of polycrystalline materials is often assumed to be isotropic owing to the random orientation of grains. When preferred orientation is present, however, the anisotropic nature of individual grains invalidates this assumption. Even if no texture exists, elastic incompatibility among grains subject to the continuous boundary introduces so-called interaction strain at the microscopic scale. Thus, the average strain components, $\left\langle\varepsilon_{i j}\right\rangle$, in the diffracting volume are the sum of (1) the homogeneous elastic strain, $\varepsilon_{i j}^{0}$, that would be observed under macro residual stress or external load, $\sigma^{0}$, (2) the average grain interaction strain, $\left\langle\varepsilon_{i j}\right\rangle^{\text {in }}$, proportional to $\varepsilon_{i j}^{0}$, and (3) the average residual elastic strain, $\left\langle\varepsilon_{i j}\right\rangle^{\mathrm{r}}$, that constrains the incompatibility owing to the inhomogeneous distribution of plastic flow, which is generally independent of external load within the elastic regime (Chidambarrao et al., 1997; Noyan \& Nguyen, 1989). In other words, the measured stress in the diffracting volume is the sum of (1) residual or applied macrostress, (2) microstress due to elastic incompatibility, and (3) microstress due to differential plastic deformation (Noyan \& Cohen, 1987).

The diffraction elastic constant, DEC (or X-ray elastic constant, XEC), needs to be employed for accurate correlation between the average local strain and the macrostress $\left(\sigma^{0}\right)$ state since the average strain measured by diffraction, $\left\langle\varepsilon_{i j}\right\rangle$, is

\footnotetext{
$\overline{\mathbf{5}}$ The $1 / e$ penetration depths for steel and aluminium are 12 and $25 \mu \mathrm{m}$, respectively, for $5.41 \mathrm{keV}(\mathrm{Cr} K \alpha)$.
}

Table 6

Effect of theoretical and experimental diffraction elastic constants from in situ neutron diffraction measurement of f.c.c. aluminium (Clausen et al., 1998).

The modulus unit is in GPa and $E_{\text {macro }}=70 \mathrm{GPa}$ for the isotropic model.

\begin{tabular}{llllll}
\hline F.c.c. aluminium reflections & 111 & 200 & 220 & 311 \\
\hline$E_{h k l}$ & 73 & 68 & 72 & 70 \\
& $\begin{array}{l}\text { Kroner model } \\
\text { ND experiment (Clausen } \\
\quad \text { et al., 1998) }\end{array}$ & 61 & 77 & 65 & 72 \\
& & & & & \\
$\sigma_{\text {measured }} / \sigma_{\text {applied }}$ & Isotropic model $[(1+v) / E]$ & 1.15 & 0.91 & 1.07 & 0.97 \\
& Kroner model $\left(S_{2} / 2\right)$ & 1.20 & 0.88 & 1.10 & 0.97 \\
\hline
\end{tabular}

not the same as the homogeneous strain, $\varepsilon_{i j}^{0}$, in general, as expressed in equation (12):

$$
\left\langle\varepsilon_{i j}\right\rangle=\varepsilon_{i j}^{0}+\left\langle\varepsilon_{i j}\right\rangle^{\text {in }}+\left\langle\varepsilon_{i j}\right\rangle^{\mathrm{r}} .
$$

The DEC can be calculated from single-crystal stiffness data (Simmons \& Wang, 1971) by using various grain interaction models, or it can be determined experimentally. If the latticeplane-dependent DECs $\left[S_{2} / 2(h k l)=\left(1+v_{h k l}\right) / E_{h k l} \quad\right.$ and $\left.S_{1}(h k l)=-v_{h k l} / E_{h k l}\right]$ are found, equation (4) can be rewritten as

$$
\left[\frac{d_{\psi \varphi}-d_{0}}{d_{0}}\right]_{h k l}=\left[\frac{S_{2}}{2} \sigma_{\varphi} \sin ^{2} \psi+S_{1}\left(\sigma_{11}+\sigma_{22}\right)\right]_{h k l} .
$$

Our main interest is how much error is introduced by the choice of reflection ( $\mathrm{Fe} 211$ and $\mathrm{Al} 222,311$ ) when using equations (5) and (10) due to the elastic anisotropy. This can be checked easily by comparing bi-axial moduli, $(1+v) / E$, with theoretically calculated DECs, $S_{2} / 2(h k l)$. However, experimentally measured DECs are often necessary because the DEC depends on the entire sample history and metallurgical state (Marion \& Cohen, 1976).

Insight on this difference can be gained by using spallation neutron diffraction (ND), since it provides longitudinal $\left(\psi_{0}=\right.$ $\left.90^{\circ}\right)$ and transverse $\left(\psi_{0}=0^{\circ}\right)$ strain simultaneously and is a convenient way of finding experimental DECs for all measured reflections. ${ }^{6}$ In situ loading ND data on bodycentered cubic (b.c.c.) ferritic steel (Daymond \& Priesmeyer, 2002) and f.c.c. aluminium (Clausen et al., 1998) have been reported. The $E_{h k l}$ and measured stresses for each reflection were calculated from those references and are listed in Tables 5 and 6. Among the anisotropic theoretical models, only the Kroner model is shown as it is known to best match experimental data (Macherauch \& Wolfstieg, 1977). Experimental DECs from ND measurements cannot be directly applied to our study, but it is informative to see differences between multiple reflections which are not usually accessible to X-ray techniques.

The differences between the isotropic and Kroner models for all Fe 211, Al 311 and $\mathrm{Al} 222$ reflections were less than or equal to 5\%; the Fe 200 and 310 peaks should not be used

\footnotetext{
${ }^{6} E_{h k l}\left(\sigma_{\text {app }} / \varepsilon_{\|, h k l}\right)$ and $v_{h k l}\left(\varepsilon_{\perp, h k l} / \varepsilon_{\|, h k l}\right)$ can be measured directly from two orthogonal strains, which is equivalent to the two-tilt method with $\psi_{0}=90^{\circ}$. However, any nonlinearity in $d$ versus $\sin ^{2} \psi$ cannot be captured in this manner.
} 
without the DEC. ND data also show that Fe 211 and Al 311 are actually a good choice because the measured stresses match well with applied (known) ones, within $3 \%{ }^{7}$ The same result for Fe 211 was confirmed by another recent set of ND data (Shrestha et al., 2015). Interestingly, much higher stresses in $\mathrm{Al} 222$ than in $\mathrm{Al} 311$ are observed using both X-rays (43\%, Fig. 13) and neutrons (23\%, Table 6) (Clausen et al., 1998). Although it is hard to draw a solid conclusion from the weak 222 intensity, this might be related to texture heterogeneity as illustrated in Fig. 9, because elastic anisotropy alone can only account for $5 \%$ of the stress. This is not surprising, as differences of $25-40 \%$ between theoretical and experimental values are often quoted in the literature (Marion \& Cohen, 1976).

In summary, if diffraction elastic constants are not used, Fe 211, $\mathrm{Al} 311$ and $\mathrm{Al} 222$ can cause about 5\% error from elastic anisotropy. $\mathrm{Al} 222$ appears to be more sensitive to the sample heterogeneity than other peaks, on top of having less intensity. The grain interaction strain, $\left\langle\varepsilon_{i j}\right\rangle^{\text {in }}$, cannot be explained solely by elastic incompatibility in a randomly oriented grain ensemble, since it should change with the surroundings of the diffracting grains in the presence of preferred orientation.

\subsection{Uncertainty from pseudo-macrostress}

Plastic deformation introduces inhomogeneous partitioning of internal strains on the macro and/or micro scale (Noyan \& Cohen, 1987). Especially when axisymmetric plastic deformation such as uniaxial tension, rolling or drawing takes place, macroscopic equilibrium is not satisfied owing to the development of pseudo-macrostress (Cullity, 1976). In such a case, the stress measured from the diffraction technique can be biased from micro residual strain, $\left\langle\varepsilon_{i j}\right\rangle^{\mathrm{r}} \neq 0$, even if interaction strains, $\left\langle\varepsilon_{i j}\right\rangle^{\text {in }}$, are taken care of by the DEC as expressed in equation (12). This is a problem for researchers interested only in macro residual stress $\left(\sigma^{0}\right)$ to evaluate engineering performance, such as fatigue life prediction. Thus, it is important to know the deformation history of the specimen under investigation.

It is unfortunate that the detailed processing history of our tested samples is not known. However, on the basis of EBSD (Fig. 2) and the XRD pattern revealing a very well known (110) texture along the vertical direction, we can speculate that plastic deformation such as drawing had taken place in our steel bar sample during the manufacturing process, leading to a pseudo-macrostress (PM stress). ${ }^{\mathbf{8}}$ Many researchers have shown that this fictitious stress is a typical phenomenon in plain carbon steel. PM stress is proportional to the amount of plastic deformation, and linearly increases up to $0.5 \%$ carbon content (Taira et al., 1974). Fe 211 shows lower stress values than Fe 310 owing to the texture development, even when DECs are used (Dolle \& Hauk, 1977; Taira et al., 1974). Our independent X-ray measurement comparing measured stresses between 1018 carbon steel (C: $0.14-0.20 \mathrm{wt} \%$ ) and

\footnotetext{
${ }^{7}$ This is observed in quasi-homogeneous materials without considerable texture, so the number is not invariant.

${ }^{8}$ The linearity of $a_{1}$ versus $\cos \alpha$ in equation (10), similar to $d$ versus $\sin ^{2} \psi$ in equation (5), was examined in all the data used in Table 3, none of which showed an oscillatory behavior.
}

1010 carbon steel (C: $0.08-0.13 \mathrm{wt} \%$ ) reveals that 1018 steel with more carbon content shows $5-25 \%$ higher stress values than 1010 steel at $25 \leq \psi_{0} \leq 45^{\circ}$, which may indicate the presence of PM stresses. Since we only know our macro applied stress, $\sigma^{0}$, stresses measured with X-rays should not be accurate for drawn carbon steel materials. The exact quantification of such an error is a difficult task.

It can be argued that the slope (measured over applied stress) depicted in Fig. 11 should not be affected because errors caused by PM stress or reflection dependency are independent of external load within the elastic regime, only causing stress offset. However, this may not be true if the micro residual strain, $\left\langle\varepsilon_{i j}\right\rangle^{\mathrm{r}}$, is affected during loading owing to the relaxation or reconfiguration of residual stress by local plastic deformation.

In summary, a part of the overall error displayed in Fig. 11 comes from the presence of pseudo-macrostress in our tested sample. These types of sample dependency should not be neglected despite the difficulty in quantifying them.

\subsection{Uncertainty from instrument calibration}

In addition to the sample effect, the instrument itself can have a significant effect on the overall uncertainty. Optical hardware components for the beam divergence, $K \beta$ filtering, alignment, software control for the peak fitting and beam center calibration are the factors determining measurement accuracy. It has been found that using a $K \beta$ filter for the $\mathrm{Cr}$ source is not an absolute requirement for high-resolution scans for a given beam size ( $2 \mathrm{~mm}$ in diameter) and short SDs $(25-45 \mathrm{~mm})$, which led to a $2.3^{\circ}$ FWHM $(2 \theta)$ for the ideal sample. The current hardware configuration and detector resolution seem good enough to provide precise measurement, but the beam center calibration can be improved as the measured stress has a strong dependency on the sample tilt angle $\left(\psi_{0}\right)$, as shown in Fig. 11. As of now, correction for the SD dependency is implemented in the calibration process, but correction for the tilt angle $\left(\psi_{0}\right)$ is not.

\subsection{Uncertainty from the measurement environment}

Factors affecting measurement accuracy have been discussed so far. Motion influencing the preset SD or $\psi_{0}$ is the major source of error in precision, but it is not consistent for each experiment. For example, in our previous field measurement with a steel tube sample, the measured stress precision was about $\pm 20 \mathrm{MPa}$ (single standard deviation) at the applied load of $200 \mathrm{MPa}$ between 35 and $45^{\circ} \psi_{0}$, i.e. about 10\% (Ramirez-Rico et al., 2016). In this in situ loading experiment with flat surface and relatively static conditions, however, the stress precision is about $\pm 6 \mathrm{MPa}$ (without sampling issues) at the same applied load within $20 \leq \psi_{0} \leq$ $48^{\circ}$, i.e. about $3 \%$. If sampled at different locations, this precision increases to $\pm 13 \mathrm{MPa}$ for the same conditions. Therefore, it is advisable to perform multiple measurements for a given set of experimental conditions to identify external and/or sampling uncertainties. Note that the precision from 
the reference powders under the most static conditions was $2 \mathrm{MPa}$ for steel.

\section{Recommended procedure for accurate stress measurement with a portable X-ray machine}

The above discussion allows us to propose a set of recommendations for successful measurements using a setup such as the one described here. The device-specific numbers are listed, but the basic protocol can be applied to any portable measurement system, regardless of detector type (image plate, $\mathrm{CCD}$ or semiconductor) or detector geometry (point detector rotation, one-dimensional linear PSD or two-dimensional).

(1) The multi-tilt $\sin ^{2} \psi$ method can be used with a small portable device as long as a sample is placed exactly at the center of rotation. However, the alignment process is time consuming, so it is not recommended if measurement speed and sample throughput are important (i.e. in a production line or manufacturing setting).

(2) The two-tilt $\sin ^{2} \psi$ method with a single exposure (at $\alpha=$ 0 and $180^{\circ}$, for example) is suggested if a sample is quasihomogeneous, i.e. no oscillation of $d$ versus $\sin ^{2} \psi$ is expected. Since no sample tilt is required, no alignment is necessary, which means that measurement is fast without much displacement error. This method yields the same results as the $\cos \alpha$ method.

(3) The $\cos \alpha$ or full-ring fitting method is preferred in the case of a small device with a two-dimensional detector because it is fast and insensitive to displacement errors, and because it utilizes a larger information volume than the two-tilt $\sin ^{2} \psi$ method. However, the linearity of $a_{1}$ versus $\cos \alpha$ needs to be checked, and the measurement accuracy is highly dependent on the beam center calibration procedure. If the beam center is not properly calibrated, the multi-tilt $\sin ^{2} \psi$ method should be considered despite its alignment difficulties, because it is controlled by the user, whereas beam center calibration is not, at least for this specific device.

(4) Regardless of the analysis method, the diffraction elastic constant and deformation history of the sample have to be considered to account for the elastic anisotropy and heterogeneity effects.

(5) For this specific device, $30 \leq \mathrm{SD} \leq 50 \mathrm{~mm}$ and $25 \leq \psi_{0} \leq$ $45^{\circ}$ are generally recommended. The best practice would be to create a map like Fig. 11 from the known stress values to account for the combined effect of factors such as DEC, pseudo-macrostress and other measurement parameters on the choice of the one single variable $\psi_{0}$ : for example, the best accuracy happens at $\psi_{0}=45^{\circ}$ for our 1018 carbon steel. If only relative values are needed for the in-line quality control, the most precise conditions $\psi_{0}=35^{\circ}$ and SD around $35-40 \mathrm{~mm}$ are recommended. Note that an accuracy calibration chart like Fig. 11 is not invariant and is subject to change by a new instrument calibration or sample conditions.

(6) The precision needs to be identified for given measurement conditions by repeating measurements at least five times, as it is affected by the external environment. If sampling statistics are needed, the measurement spot should be varied at each measurement.

\section{Conclusions}

Owing to the growing interest in portable X-ray stress analyzers utilizing area detectors, the precision and accuracy of stress measurements have been rigorously tested by parameterizing the sample-to-detector distance (SD) and instrument tilt angle against the sample normal $\left(\psi_{0}\right)$. The intrinsic instrument precision was measured in ferrite reference powders, while 1018 carbon steel bar samples were used for accuracy tests in situ under tensile loading.

The measurement precision can be as good as $2 \mathrm{MPa}$ (for $\mathrm{Fe}$ powders) in a very controlled environment, whereas precisions of 13 and $6 \mathrm{MPa}$ (for the steel solid bar) were obtained for in situ loading experiments at $200 \mathrm{MPa}$, depending on whether sampling statistics were or were not accounted for, respectively. In field conditions with vibrational background noise, the precision was about $20 \mathrm{MPa}$. Consequently, we recommend performing multiple measurements at the same and different sample locations to quantify uncertainties arising from external sources and sample heterogeneity.

Accuracy is determined by many factors, such as instrumental parameters (beam center alignment and calibration), measurement parameters (SD and $\psi_{0}$ ), sample conditions (deformation history and texture) and physical properties (elastic and plastic anisotropy). In this specific device, tilt angle $\left(\psi_{0}\right)$ turned out to be the most sensitive parameter affecting stress accuracy and precision, probably associated with beam center determination, which is typically not customizable by users. The appropriate ranges of SD and $\psi_{0}$ were found for the accurate stress assessment from the 1018 steel bar sample. From the practical point of view, it would be best to create a material-specific accuracy calibration chart as a function of a single parameter $\left(\psi_{0}\right)$ owing to the complexity of the individual factor corrections by applying the diffraction elastic constant or subtracting pseudo-macrostress. However, if relative values are important or sufficient, measurement can be done with the suggested settings, $\psi_{0}=35^{\circ}$ and SD around $35-40 \mathrm{~mm}$, where the best precision is found.

The portable X-ray machine using an image plate is an efficient stress measurement tool for quasi isotropic and homogeneous materials when the linearity of the strain component versus $\cos \alpha$ (or $\sin ^{2} \psi$ ) is valid. However, for materials with significant elastic anisotropy and heterogeneity which possess microstress and/or pseudo-macrostress, the simple stress models included in the manufacturer's software for rapid analysis cannot be used and more advanced mathematical treatments or calibration are required.

\section{Acknowledgements}

The authors appreciate hardware and software installation from the manufacturer of the portable X-ray device. We acknowledge Adrian Brügger for providing tensile loading facilities at the Robert W. Carleton Strength of Materials 
Laboratory in Columbia University, and his colleagues, Liming $\mathrm{Li}$ and Janelle A. Mill, for their excellent support during the tensile loading tests. We also thank Adrian Chitu for his help with optical microscope and EBSD measurements. JRR acknowledges support in the form of a travel grant from the V Plan Propio, Universidad de Sevilla. Fruitful discussions and input from Professor I. C. Noyan are gratefully acknowledged.

\section{References}

Amemiya, Y. \& Miyahara, J. (1988). Nature, 336, 89-90.

Araki, H. (1989). Rigaku J. 6, 34-42.

Bolstad, D. A. (1967). SAE Technical Paper 670152, pp. 1-4. SAE International, Detroit, Michigan, USA.

Brauss, M. E., Gorveatte, G. V. \& Porter, J. F. (1996). Proc. SPIE, 2944, 307-317.

Chen, C. M. \& Kovacevic, R. (2003). Int. J. Mach. Tools Manuf. 43, 1319-1326.

Chidambarrao, D., Song, Y. \& Noyan, I. (1997). Met. Mater. Trans. A, 28, 2515-2525.

Clausen, B., Lee, S. Y., Ustundag, E., Aydiner, C. C., Conner, R. D. \& Bourke, M. A. M. (2003). Scr. Mater. 49, 123-128.

Clausen, B., Leffers, T. \& Lorentzen, T. (2003). Acta Mater. 51, 61816188.

Clausen, B., Lorentzen, T. \& Leffers, T. (1998). Acta Mater. 46, 30873098.

Cullity, B. D. (1976). Adv. X-ray Anal. 20, 259-271.

Daymond, M. R. \& Priesmeyer, H. G. (2002). Acta Mater. 50, 16131626.

Dölle, H. (1979). J. Appl. Cryst. 12, 489-501.

Dolle, H. \& Hauk, V. (1977). Z. Metallkd. 68, 725-728.

Eathough, M. O., Rodriguez, M. A., Blanton, T. N. \& Tissot, R. G. (1999). Adv. X-ray Anal. 41, 319-326.

Farrell, S. P. (2010). Powder Diffr. 25, 119-124.

Ganesh, P., Kumar, H., Kaul, R. \& Kukreja, L. M. (2013). Surf. Eng. 29, 600-607.

Hashin, Z. \& Shtrikman, S. (1962). J. Mech. Phys. Solids, 10, 335-342.

He, B. B. (2011). Two-Dimensional X-ray Diffraction. Hoboken: John Wiley and Sons.

Homicz, R. (1967). SAE Technical Paper 0148-7191. SAE International, Detroit, Michigan, USA.

James, M. R. (1977). Proceedings of the ARPA/AFML Review of Progress in Quantitative NDE, pp. 93-98. Air Force Materials Laboratory, Air Force Wright Aeronautical Laboratories, Air Force Systems Command, Wright-Patterson Air Force Base, Ohio 45433, USA.
James, M. R. \& Cohen, J. B. (1978a). J. Test. Eval. 6, 91-97.

James, M. R. \& Cohen, J. B. (1978b). Treatise Mater. Sci. Technol. 19, $1-118$.

Kampfe, A., Kampfe, B., Goldenbogen, S., Eigenmann, B., Macherauch, E. \& Lohe, D. (2000). Adv. X-ray Anal. 43, 54-65.

Kiss, M. Z., Sayers, D. E. \& Zhong, Z. (2002). Nucl. Instrum. Methods Phys. Res. Sect. A, 491, 280-290.

Kroner, E. (1958). Z. Phys. 151, 504-518.

Ling, J. \& Lee, S.-Y. (2015). Adv. X-ray Anal. 59, 153-161.

Macherauch, E. \& Wolfstieg, U. (1977). Mater. Sci. Eng. 30, 1-13.

Marion, R. H. \& Cohen, J. B. (1976). Adv. X-ray Anal. 20, 355-367.

Miyahara, J., Takahashi, K., Amemiya, Y., Kamiya, N. \& Satow, Y. (1986). Nucl. Instrum. Methods Phys. Res. Sect. A, 246, 572-578.

Miyazaki, T. \& Sasaki, T. (2016). J. Appl. Cryst. 49, 426-432.

Monin, V. I., Assis, J. T., Pereira, F. R., Gurova, T. \& Teodósio, J. (2003). Mater. Sci. Forum, 426-432, 4531-4534.

Monin, V., Teodosio, J. \& Gurova, T. (2000). Adv. X-ray Anal. 43, 6671.

Noyan, I. C. \& Cohen, J. B. (1987). Residual Stress. New York: Springer-Verlag.

Noyan, I. \& Nguyen, L. (1989). Adv. X-ray Anal. 32, 355-364.

Öztürk, H., Yan, H., Hill, J. P. \& Noyan, I. C. (2014). J. Appl. Cryst. 47, 1016-1025.

Öztürk, H., Yan, H., Hill, J. P. \& Noyan, I. C. (2015). J. Appl. Cryst. 48 , 1212-1227.

Ramirez-Rico, J., Lee, S.-Y., Ling, J. \& Noyan, I. C. (2016). J. Mater. Sci. 51, 5343-5355.

Ruud, C., DiMascio, P. \& Snoha, D. (1984). Adv. X-ray Anal. 27, $273-$ 283.

Sasaki, T., Hirose, Y., Sasaki, K. \& Yasukawa, S. (1997). Adv. X-ray Anal. 40, 588-594.

Sasaki, T. \& Kobayashi, Y. (2009). Adv. X-ray Anal. 52, 248-255.

Sasaki, T., Maruyama, Y., Ohba, H. \& Ejiri, S. (2014). J. Instrum. 9, C07006.

Shrestha, T., Charit, I. \& Potirniche, G. (2015). J. Mater. Eng. Perform. 24, 4710-4720.

Simmons, G. \& Wang, H. (1971). Single Crystal Elastic Constants and Calculated Aggregate Properties: A Handbook, 2nd ed. Cambridge: MIT Press.

Sonoda, M., Takano, M., Miyahara, J. \& Kato, H. (1983). Radiology, 148, 833-838.

Taira, S., Hayashi, K. \& Ozawa, S. (1974). Proceedings of the Symposium on Mechanical Behavior of Materials, pp. 287-295. Kyoto: Society of Materials Science.

Taira, S. \& Tanaka, K. (1979). Trans. Iron Steel Inst. Jpn, 19, 411-418.

Taira, S., Tanaka, K. \& Yamazaki, T. (1978). J. Soc. Mater. Sci. Jpn, 27, 251-256.

Yoshioka, Y. \& Ohya, S. (1992). Adv. X-ray Anal. 35, 537-543. 